Itinéraires Itinéraires

Littérature, textes, cultures

\title{
Le journal au seuil de l'intimité
}

\section{Philippe Lejeune}

\section{OpenEdition}

\section{Journals}

Édition électronique

URL : http://journals.openedition.org/itineraires/610

DOI : 10.4000/itineraires.610

ISSN : 2427-920X

Éditeur

Pléiade

Édition imprimée

Date de publication : 1 décembre 2009

Pagination : 75-90

ISBN : 978-2-296-10791-5

ISSN : 2100-1340

Référence électronique

Philippe Lejeune, «Le journal au seuil de l'intimité », Itinéraires [En ligne], 2009-4 | 2009, mis en ligne le 24 juillet 2014, consulté le 19 avril 2019. URL : http://journals.openedition.org/itineraires/610 ; DOI

10.4000/itineraires.610

Ce document a été généré automatiquement le 19 avril 2019

\section{(c) (i) (9)}

Itinéraires est mis à disposition selon les termes de la licence Creative Commons Attribution - Pas d'Utilisation Commerciale - Pas de Modification 4.0 International. 


\title{
Le journal au seuil de l'intimité
}

\author{
Philippe Lejeune
}

$1 \quad \mathrm{Au} \mathrm{XVIII}^{\mathrm{e}}$ siècle, le journal est entré dans l'intimité... à reculons. Ou bien, disons que l'intimité est venue habiter le journal... à regret. Nous sommes tellement habitués à l'expression « journal intime » qu'il nous est difficile de réaliser que ce qui est pour nous un pléonasme a pu être, au XVIII ${ }^{\mathrm{e}}$ siècle, un oxymore, une contradiction dans les termes : le journal était alors fait pour tout, sauf pour l'intime! C'était une écriture, sinon publique, du moins partageable, ouverte à la lecture d'autrui, et parfois à son écriture. Il était fait pour être consulté et transmis. C'était une écriture, sinon factuelle, du moins objective, précise, mémorielle, où le commentaire, l'interprétation, l'affectivité n'avaient pas leur place.

2 Les modèles du journal étaient le livre de comptes, la chronique historique, le livre de raison familial, le livre de bord et la relation de voyage. Soit par leur auteur (pour le livre de raison), soit par leur objet (comptes, histoire, voyage), les journaux étaient fondamentalement sociaux. Au début du XVII ${ }^{\mathrm{e}}$ siècle, on avait découvert la possibilité de coupler la périodicité de l'écriture avec celle d'une publication: c'est l'invention de la presse périodique. Ces publications, doublement collectives (par leur production et leur lecture immédiate), même quand elles n'étaient pas quotidiennes (le premier quotidien français, Le Journal de Paris, a paru seulement en 1777), comportaient parfois dans leur titre le mot «journal», et même si ce n'était pas le cas, portaient ce nom générique, renforçant l'image sociale et factuelle liée à ce mot. « Faire un journal », c'était donc faire un compte rendu, rapport ou récit précis, détaillé, de faits ou d'occupations, en suivant l'ordre du temps. Rien n'empêchait, en principe, que l'objet rapporté fût un état d'âme, et que l'écriture n'eût d'autre destinataire que son auteur. Mais dans la pratique, en France, il semble que jamais une idée aussi saugrenue n'était venue à personne. On en a une preuve indirecte en lisant La Conversation avec soi-même (1751, réédité en 1760 et 1762) du marquis Caraccioli : dans ce manuel de "développement personnel», comme nous dirions aujourd'hui, jamais l'auteur n'émet l'idée que cette conversation intime pourrait passer par l'écriture, laisser des traces et permettre un dialogue dans le temps. 
3 Je voudrais en faire ici la démonstration en analysant le métadiscours d'un journal particulier, dont le début (1771-1779) vient d'être publié, celui de Louis-François Guiguer, baron de Prangins. Il écrit dans son journal, le 17 avril 1777: «Mais basta. Ceci est un journal ", pour mettre un terme à l'expression trop nette de tendres sentiments qui le poussaient vers sa « cousine » Matilda. Un journal, c'est destiné à noter les faits, pas une déclaration d'amour! Mais Louis-François est en pleine contradiction : car il a eu envie de le faire, et il ne s'est arrêté, honteux de son expansion, que lorsqu'il a été trop tard. Et c'est en termes assez familiers (basta) qu'il s'est reproché son incursion dans l'intime... Le vent est en train de tourner, même si c'est lentement.

Qui est Louis-François Guiguer? Un homme de vingt-neuf ans qui, en 1771, à la mort de son père, s'installe au château de Prangins, près de Genève, au bord du lac. D'une certaine manière, le journal qu'il commence alors est le journal du château autant que le sien. Mais il va s'approprier le château, et le journal avec. Le volume qui vient d'être publié ${ }^{1}$ reproduit les trois premiers des sept volumes qui nous conduiront de son installation à Prangins jusqu'à sa mort (1786). La période ici couverte va de 1771 à 1779, à la veille de la naissance de son premier enfant.Elle se divise elle-même en trois phases, qui correspondent à la fois à trois étapes de sa vie relationnelle, et à trois systèmes de rédaction.

5 En effet, de qui est-ce le journal? La question se pose: trois personnes différentes y écrivent, au cours de ces années, et, y écrivant, ont été susceptibles de le lire. Ce n'est pas seulement le journal d'un individu, mais celui d'une petite cellule sociale: un couple d'amis, puis un couple de fiancés, puis un couple d'époux. On parle de «symbiose », il faudrait inventer «syngraphie» ou «syngraphose» pour désigner cette écriture partagée.

6 Impossible de raconter l'histoire des personnes sans passer, ici, par celle de leur écriture. Je vais la résumer à grands traits, en montrant le conflit entre une pratique sociale de notation factuelle et une pulsion d'expression personnelle qui fait peu à peu craquer l'ancien modèle.

\section{Première phase : du 31 mars 1771 au 21 juillet 1776 (p. 31-297)}

7 Pendant ces cinq années, Louis-François tient son journal... sans l'écrire! Le texte est entièrement de la main de son ami et bras droit, Christophe-Daniel Renz (1742-1826), juriste, et musicien, d'un an plus jeune que lui, qui partage sa vie au château de Prangins.

Qui est responsable de ce texte ? La répartition des rôles est difficile à saisir, parce qu'elle n'est pas constante, et que la connivence entre les deux amis semble totale. Disons, entre parenthèses, que l'un des charmes de la lecture de ce journal tient à l'harmonie des relations amicales puis amoureuses qu'il reflète. Renz tient donc constamment la plume : écrit-il sous la dictée de Louis-François, ou de sa propre initiative? Sans doute les deux, en proportions variables selon les circonstances. Leur vie est tellement commune que le journal est presque toujours écrit en "nous». Ils s'appellent eux-mêmes «les journalistes », mais distinguent parfois « le journaliste écrivain » ou « l'écrivain » (Renz) de l'autre, parlant alors d'eux-mêmes à la troisième personne. La situation se corse, et l'énonciation se complique, quand leur sort, pour quelques heures, se sépare, et que la journée demande deux récits distincts. De mai à juillet 1772, par exemple, une dizaine de 
fois les «journalistes" se livrent par nécessité, mais aussi par jeu, à de pittoresques variations sur renonciation. 14 mai : «Un des journalistes rend compte à l'autre. Je suis parti à $7 \mathrm{~h} 1 / 2$ pour Sécheron [...]» (p. 122). Suit le récit de la journée... duquel des deux ? Je penche pour Renz, mais l'éditeur, indécis sans doute, ne m'aide guère: pas de note pour trancher. L'essentiel est ailleurs : nous sommes indécis, mais pas eux! Ils savent qui est qui ! Et le journal, moins par son contenu que par sa forme, entre dans l'espace, sinon intime, du moins confidentiel, de la correspondance. Nous assistons à leur dialogue, qu'ils se (et nous) donnent en spectacle. 22 mai, le jeu continue: "Moi (qui ne suis pas l'écrivain), je suis parti seul en voiture pour Aubonne [...]. Moi l'écrivain, j'ai relevé le dessin de la place de l'étoile et fait le devis pour clôture le long du grand chemin [...]» (p. 130). La première formule est drôle, renvoyant à l'autoréférence de la parole citée, non à celle de l'écriture que nous lisons... 28 mai, nouvelle devinette : «Un de nous : départ à 7 heures à pied au devant de Madame de Prangins [...]. Quant à l'autre, il n'est point allé en maison de deuil, mais bien chez nos voisins de Vinzel [...]» (p. 135). 7 juin, rebelote : « Comme ma journée a commencé à quatre heures du matin, je dois parler le premier. J’ai vu les deux ministres allemands, entendu deux sermons, dîné chez Monsieur Delarive [...]. Je n'ai pas si bien employé ma journée [...] » (p. 142-143). 17 juin, taquinerie : «J'ai passé la nuit au bal. Il n'est pas besoin de dire qui de nous deux prend la parole puisqu'il s'agit de bal » (p. 151). C'est Renz, nous dit une note. À remarquer l'expression " prend la parole » pour désigner, cette fois, l'écriture! Nous sommes devant une sorte de «bulle» gémellaire. Cette co-énonciation, qui produit un effet de conversation familière, exclut cependant l'intimité, c'est-à-dire l'expression de sentiments individuels - par exemple amoureux. Ce n'est donc pas un hasard si le journal tenu par les deux amis s'est arrêté pile au moment de l'arrivée à Prangins de Matilda Cleveland, à la mi-juillet 1776.

9 Au long de ces cinq années, le rythme de ce journal commun va évoluer. Il est tenu de manière strictement quotidienne pendant quatre mois, du 31 mars au 4 août 1771 (p. 31-105). Puis dix-huit pages sont laissées blanches (pour un éventuel rattrapage, qui n'a pas été fait) et le journal reprend le 22 avril 1772, après huit mois et demi d'interruption. Les deux hommes sont alors à Montpellier, et cette reprise du journal coïncide avec leur voyage de retour à Prangins. Ce nouveau journal est d'abord lui aussi quotidien, mais au bout de quatre mois, à partir du 15 septembre 1772, un autre rythme est adopté : désormais, il est tenu avec des écarts de plusieurs jours, qui ont tendance à augmenter. L'habitude est prise, chaque fois qu'on reprend la plume, de résumer plus ou moins ce qui s'est passé depuis l'entrée précédente. Un dernier hommage est rendu à la continuité trahie : les entrées sont coiffées par deux dates associées, celle du début de la période rattrapée et celle du rattrapage : «mercredi 7 - mardi 13 ». Il arrive que le retard soit comblé sous la forme d'un «jour à jour» détaillé, mais il est souvent survolé de manière synthétique et parfois désinvolte. Cette continuité élastique sera fidèlement (si je puis dire !) respectée pendant quatre ans, jusqu'à ce que Renz mette fin, sans explication, à sa fonction de scribe. Le passage de la régularité à l'irrégularité est un premier décrochage par rapport au modèle. Il suppose l'exercice d'un libre arbitre, et des choix qui doivent être justifiés, comme on le verra ci-dessous.

Quels sont les champs thématiques de ce journal? On le constate facilement à la lecture, et nos " journalistes » le souligneront en s'imposant des limites ou en s'excusant de les avoir transgressées. Il y a trois champs principaux. On lit, entrelacés :

- un journal " professionnel » de la gestion du château et de ses domaines (c'est le côté livre de raison: travaux, aménagements, plantations, négociations, inspections, gestion du 
personnel, etc. - mais par ailleurs un journal plus technique est tenu à partir de 1773 sur un autre livre : «Ouverture d'après un ancien projet d'un livre pour la régie et les comptes de la terre » (27 janvier 1773, p. 213);

- un journal « social » des visites reçues et rendues, des fêtes ou spectacles donnés, des lettres, des rencontres (c'est le côté « mémoires » ou « chronique »);

- un journal « intellectuel » des livres lus, dont le contenu est résumé et l'intérêt évalué.

11 Louis-François et son ami mènent l'existence de notables occupés à faire valoir leurs terres, ils ont une vie sociale très active et participent à la vie intellectuelle de leur époque.

Quand ils commentent leur pratique, nos journalistes abordent trois sujets qui mettent en question les limites du journal : la digression, la censure, la négligence.

\section{La digression}

Une seule fois, le journaliste se laisse aller à des développements qu'il juge étrangers à la pratique du journal. Le 12 mai 1771, la maladie de Monsieur de Sturler lui donne occasion de se lancer dans une méditation philosophique sur la douleur et la mort, qu'il termine ainsi: "Après cette digression involontaire, nous revenons au journal» (p.72). Commenter l'expérience n'est donc pas la fonction du journal, qui doit s'en tenir aux faits. Monsieur de Sturler meurt le lendemain: bref hommage funèbre, nouvelle méditation philosophique et nouveau repentir, cette fois plus développé :

Revenons-en au journal: si les actions se succédaient aussi rapidement que les idées, il serait plus que rempli de faits auxquels nous ajoutons de l'importance; mais le détail de la vie privée nous paraît minutieux. Brisons-là pour éviter une troisième digression! (p. 73)

J'avoue peiner à suivre l'expression assez confuse de ce regret, mais j'en retiens l'idée qu'il ne faut pas accorder d'importance (dans le journal) à ce à quoi on accorde de l'importance (dans la vie), et qu'une méditation sur la mort d'un proche appartient au « détail de la vie privée » qui y serait «minutieux ». Il ressort de tout cela que le journal n'est pas le lieu pour exprimer des émotions ou des méditations. Mais il en ressort aussi que Louis-François a envie de les exprimer! Du coup, il a tendance à «détourner » le journal à cette fin. On le prendra plusieurs fois la main dans le sac de digressions sans repentir. Le 20 juillet 1771, il commente longuement sa lecture de l'Histoire naturelle de Pline et termine en s'absolvant: «Une digression qui passe de l'histoire naturelle à l'histoire morale de l'homme ne change pas de matière » (p. 103). L'année suivante, un nouveau deuil, la mort du fils de Monsieur Noël, occasionne une longue tirade dont il ne s'excuse plus du tout (26 juillet 1772, p. 172). D'autre part, une zone du journal échappe à cette exclusion de la réflexion: les comptes rendus de lecture, qui donnent l'occasion d'évaluer ou de critiquer les livres lus.

Pas plus que l'expression de la pensée ou de la méditation, le sentiment n'a sa place dans un journal, il n'est pas « de son ressort ». D'où cette conséquence paradoxale : plus la vie est passionnée, plus le journal, qui ne peut rapporter que des faits, sera vide! En témoigne ce passage, à l'expression assez contournée, mais qui exprime bien une forme de regret :

15 août 1772 - Espérance reçue de Monsieur de Saint-Saphorin qui désire de se fixer à Aubonne. Nous ne nous quittons point le reste de la journée et rien ne se passe qui soit du ressort du journal, car il peut fort bien arriver que la journée soit bien remplie et le journal très dénué : c'est que le sentiment occupe beaucoup mieux la 
vue que les actions qui n'ont pas le sentiment pour moteur et qui pourtant sont nécessaires et tirent de cette nécessité même leur importance pour nous. (p. 180)

\section{La censure}

15 Par deux fois, le journaliste fait une chose étonnante : il explicite la règle de censure qui gouverne, selon lui, la pratique du journal. S'il en prend conscience au point de l'exprimer, c'est qu'il a envie de la violer, et de retrouver sa liberté de parole. Sa plume le démange! Mais dans un journal, ça ne se fait pas!-J'avoue, à cause de cette discrétion même, ne pas bien comprendre de quoi il s'agit, mais voici ce qu'il écrit le 30 juillet 1772 : "Nouvelles reçues de Saint-Saphorin par Madame de Ribaupierre, à la Lignière. Reproches sur l'inutilité de nos projets du voyage de Saint-Saphorin. Les reproches devraient paraître, d'après le journal, être fondés ; mais le journal ne dit pas tout... » (p. 174). Il se défend donc, auprès d'un éventuel lecteur (un proche qui lirait le journal ?), en révélant que le journal ne révèle pas tout ! De la même manière, le 29 septembre 1772, après avoir nommé plusieurs personnes venues en visite à Prangins, il conclut brusquement : « Les gens que l'on a chez soi ne doivent jamais être soumis à la censure et aux portraits. Les éloges seraient fades et la critique indiscrète » (p. 195). Cette discrétion, dictée par l'hospitalité, s'applique au bien comme au mal. Malignement, on pensera qu'il s'agit ici plutôt du mal. En tout cas, le journaliste souffre de cette limite, il se rappelle luimême au respect des règles de bienséance.

\section{La négligence}

16 «La négligence de l'écrivain est cause de la lacune qu'il y a dans l'histoire de ces quatre jours. Voici ce dont il se souvient»(23-26 juillet 1772, p. 103). Assez souvent, le journaliste se plaint de ne pas arriver à rendre compte de sa vie. Il se tourne et se retourne dans une position inconfortable. Plus la vie est intéressante, moins on a le temps de la noter! Et puis le journal, spécialisé dans le factuel, apparaît comme une sorte de pensum absurde. Mais on ne se résout pas à le lâcher, on passe son temps à essayer de le « remettre au niveau» (17 octobre 1774, p. 255). Peut-être rêve-t-on d'une autre forme d'écriture? Je vais abréger cette litanie d'excuses en ne citant que les plus révélatrices de la crise.

17 Juin 1773 : il y a théâtre à Nyon pendant tout le mois, Prangins sert de base arrière, nos amis s'activent, cessent leurs autres occupations, ils sont passionnés. Moralité : «Nous renonçons à chercher les détails de ce que nous avons fait pendant ce temps de dissipation que nous ne regrettons ni pour l'avoir eu, ni pour l'avoir perdu» (p. 223). Juillet 1773: « Encore une quinzaine indifférente puisque tout détail en est omis » (p. 224). Avril 1774:

La construction d'un théâtre, les décorations, les répétitions, les invitations, l'illumination, l'habillement et le costume, etc., etc. ont tellement rempli ou, si vous voulez, fait un si grand vide dans nos journées qu'il ne reste rien dans la mémoire et qu'il ne passera rien à la postérité par ce journal que le grand événement du 27 avril 1774, jour auquel on a donné sur le théâtre de Prangins devant une assemblée respectable, avec un succès brillant, l'inimitable pièce de l'incomparable Molière, Le Misanthrope [...]. (p. 246)

- Sachez d'ailleurs que Renz était Philinte et Louis-François Alceste ! Mai-juin 1774: «Plus les deux dates sont éloignées, plus il faut que le journal soit court» (p. 248). Février-mars 1775 : « Le vide des événements ne suppose pas un vide d'occupations » (p. 269). Mars-avril 
1775 : «Les grandes lacunes ne peuvent qu'être recouvertes et non remplies» (p. 272). Décembre 1775 : «Les études vont leur train et font tort au journal auquel nous promettons plus de suite» (p. 286). Janvier 1776: « Le froid, le dormir et l'entretien de nos hôtes ont tellement ôté chaque jour le quart d'heure destiné au journal qu'au moins cette lacune-ci a une excuse vraisemblable » (p. 289). Etc.

Il y a donc un vrai désir de tenir un journal, mais un embarras devant un genre trop factuel, qui ne vaut pas qu'on y perde un temps mieux occupé à vivre. Peut-être est-ce pour cela que Renz lâchera la plume en juillet 1776. Mais, plus sûrement encore, c'est parce que, sur le théâtre de Prangins, la distribution aura changé.

\section{Deuxième phase : du 24 juillet 1776 au 2 août 1778 (p. 298-441)}

C'est la période pendant laquelle Louis-François va être seul aux commandes du journal, sans délégation de plume (ou presque). Le changement d'écriture est spectaculaire : celle de Renz était régulière et presque calligraphique, celle de Louis-François irrégulière et plus négligée - plus expressive aussi.

Pour comprendre la morphologie compliquée du journal, il faut connaître l'histoire. En juillet 1776 arrivent à Prangins en visite de longue durée une tante par alliance anglaise et deux de ses nièces, Celina et Matilda Cleveland. Matilda est « dans l'âge où à peine on finit de grandir », elle a dix-huit ans. Au bout de quelques jours, on ne s'appelle déjà plus " Monsieur » ou " Mademoiselle », mais cousin et cousine. Et très vite c'est, entre LouisFrançois et Matilda, l'amour. Les dames anglaises passent tout l'automne et l'hiver à Prangins, et au printemps 1777, on part tous ensemble (avec Renz) pour un voyage en Italie, qui durera six mois, pendant lequel Celina, l'aînée, trouvera à se marier avec le consul anglais à Livourne. Retour à Prangins fin septembre 1777. Matilda et sa tante quittent finalement le château en novembre, séjournent à Paris, rentrent à Londres. Pour Louis-François, triste hiver à Prangins : au printemps 1778, il rejoint Matilda à Londres, l'épouse et revient avec elle à Prangins en août 1778.

21 L'arrivée des dames anglaises en 1776 a eu pour conséquence l'interruption du journal, et la fin du "secrétariat » assuré par l'ami Renz. Six mois de silence total. Il semble y avoir une proportion inverse entre l'importance des événements, et la tenue du journal. Plus la vie est pleine, plus le journal est vide. Louis-François formulera lui-même cette loi, un peu plus tard. Le 25 avril 1778, partant pour l'Angleterre avec l'intention d'épouser Matilda, il laisse son journal à Prangins et lui dit au revoir en ces termes : «Je solde ici mon journal jusqu'au retour quoique peu rempli au milieu du temps de ma vie où ma tête est le plus remplie » (p. 441). La tête - et le cœur! Le paradoxe, c'est que cet échec du journal, il le confie... à son journal. Sans qu'il soit capable de l'expliciter, il y a, derrière la constatation de cette absurdité, le désir d'une autre forme d'expression, dont le pauvre journal, tel qu'on le pratique à l'époque, est incapable.

La preuve en est qu'au bout de six mois d'idylle, le $1^{\text {er }}$ janvier 1777 , Louis-François se ressaisit, désolé d'avoir interrompu un journal, qui, si pauvre fût-il, aurait jalonné sa mémoire. Il décide de le reprendre, mais aussi de rattraper les six mois de silence. Comment faire? En piochant... dans le journal de Matilda! Celui-ci, très factuel sans doute, et dont la consultation semble libre, lui fournit la matière à un substitut... dont il 
admet lui-même qu'il est un peu bâclé. Voici en quels termes désabusés il évalue ce « rattrapage »:

28 janvier 1777 - J'ai fini de recueillir des notes anglaises assez de circonstances pour remplir l'espace laissé vide dans le journal depuis l'arrivée jusqu'au commencement de cette présente année. L'exactitude ne paraît pas être décente dans la relation peu suivie de quelques observations qui n'ont guère plus d'importance que les événements historiques des gazettes. Le temps qu'il faudrait pour se rappeler les journées passées me détermine toujours à les laisser échapper. (p. 311)

On retrouve ici sa complainte sur la pauvreté du journal, et la perte de temps qu'il occasionne. Mais alors, pourquoi continue-t-il ?

Le journal de rattrapage, puis le journal régulier du $1^{\mathrm{er}}$ janvier au 20 mars 1777 (départ pour l'Italie), sont très différents de la chronique tenue sous le règne de Renz: LouisFrançois flirte avec le lyrisme, il balbutie maladroitement des émotions dans les interstices d'un tissu de données factuelles. Il voudrait dire quelque chose, mais se retient. Une des raisons de cette retenue est le caractère ouvert, public, de ce journal. Il cultive donc une rhétorique de l'allusion. Exemples :

28 juillet 1776 - Les leçons de clavecin ont commencé pour Cousine dont les succès ont dès ce jour-là été prévus et prédits. Il en sera assez souvent reparlé dans la suite du journal. (p. 298)

30 juillet 1776 - Course à Fernex en deux voitures [...]. Mais de tout cela, le retour par une belle lune, qui nous a reposé du grand soleil, est ce qui m'a fait de beaucoup le plus grand plaisir ou, pour mieux dire, le seul qui vaille que le souvenir en soit fixé. Nous avons déjà depuis lors pris assez constamment le nom de cousin à la place de Monsieur et Mademoiselle. (p. 298)

[Le 20 août, il a fait une chute en voiture en conduisant...]

26 août 1776 - Mon séjour au lit depuis la chute est adouci par l'assiduité dont j'aime mieux me souvenir que me vanter. Ce sont les Anglaises qui m'ont si bien gardé que... (p. 300)

11 septembre 1776 - Dîné à Changins. Je voudrais bien savoir pourquoi j'ai de ce dîner un souvenir si détaillé et si présent. (p. 301)

29 décembre 1776 - Départ pour un bal ; il me reste une cousine et cela vaut bien le bal que je leur abandonne. (p. 307)

Le journal recommencé le $1^{\mathrm{er}}$ janvier 1777 titube, lui, entre des plaintes contradictoires, mais complémentaires. Ou bien le journal est taxé d'incapacité, tout juste bon à noter des faits, il est incapable de capter les émotions, de dire le bonheur :

5-6 janvier 1777 - Aucuns jours ne passent mieux pour moi que ceux qui fournissent peu au journal puisque notre uniformité et notre solitude n'est pas interrompue. (p. 309)

Ou bien Louis-François cède au plaisir de dire qu'il a vibré devant un paysage, mais il s'en excuse en attaquant les Béotiens qui pourraient trouver cela ridicule :

11-15 janvier 1777 - Deux très beaux jours au milieu de l'hiver : l'un d'eux fournit deux heures à une promenade en cabriolet: la campagne était variée et vivante. Ceux qui ne sentent point traitent d'images poétiques tout ce qu'expriment, dans une simple description, mais fine et détaillée, ceux que touchent les beautés frappantes et les charmes multipliés de la Nature. (p. 310)

Ce qui «multiplie » les charmes de la nature, sans doute est-ce la présence de Matilda dans le cabriolet. L'argumentation peut ici sembler étrange, car la description « fine et détaillée » qu'il justifie en attaquant les prosaïques... cette description n'est pas dans le journal ! Il se justifie de quelque chose qu'il n'ose faire : un journal... poétique ! Jusqu'au départ pour l'Italie, son journal va se traîner dans l'insignifiant. Il nous fait savoir de temps en temps qu'il est heureux, mais qu'il ne saurait le dire qu'en disant qu'il est 
incapable de le dire : «19-21 février 1777 - Rien, sinon tranquillité et petites occupations pour lesquelles les journées ne sont pas assez longues » (p. 314).

Le voyage en Italie (p. 319-408), qui les mènera jusqu'à Florence et Pise, est tenu sur un livre spécial, d'un format légèrement plus grand. Le journal de voyage est un genre dans lequel Louis-François se sent beaucoup plus à l'aise. En voyage, les impressions sont ellesmêmes des événements, et l'on peut être personnel sans toucher à l'intimité. Dès qu'en octobre 1776 la décision a été prise de partir, il a remarqué : «Cela sans doute doit fournir un beau sujet au journal » (p. 305). Le danger, c'est de plagier les guides, de répéter ce qui est décrit partout. Le 24 mars 1777 à peine arrivé à Chambéry, il le signale :

Chambéry est connu à tout le monde; ainsi nous pourrions étendre la relation autant qu'il nous plairait. Mais qu'il soit mis pour règle, une fois pour toutes, que c'est notre voyage dont nous voulons parler, et non de l'article d'un dictionnaire politique, historique, etc. (p. 327)

Une piqûre de rappel à Turin, le 29 mars : « Je ne suis point moins sensible à la sculpture, à la peinture, à l'architecture et au génie qui a souvent inventé et conduit de grandes entreprises en ce genre parce que je n'en parlerai pas toujours » (p. 332). Il ne fera donc aucune description systématique, mais parlera librement de ses impressions et des incidents du voyage. Jusqu'où ira cette liberté ? Elle ne devra pas, à l'autre extrême, se permettre la confidence intime, mais ne pourra s'empêcher de la frôler.

Les limites, de ce côté, sont posées dès le départ, et leur raison est donnée : ce journal, ouvert à la lecture des compagnons de voyage, peut se permettre des allusions, des douceurs, mais rien de vraiment intime. La «publicité » du journal est réciproque : à deux reprises, on verra Louis-François critiquer le journal de Matilda (10 juillet et 3 août, p. 385 et 389). C'est pourquoi il renonce à faire le portrait des trois voyageuses :

21 mars 1777 - Comme j'écris la relation sous les yeux des dames, je n'ose faire pour elles ce qui sans doute se trouverait fort bien à la tête du récit d'un voyage entrepris pour elles : leur portrait. D'ailleurs, je serais bien assuré, à la vérité, de ne pas flatter par politesse; mais qui voudrait exiger de l'amitié de se dégager des préventions en ôterait tout le charme. (p. 326)

Cela ne va pas l'empêcher de rôder autour du madrigal :

23 mars 1777 - Nous dînons. Les dames nous accordent pour troisième dans notre voiture ma cousine Matilda. Cela détourne un peu des observations sur ce qui se passe hors de la voiture, mais le plaisir du voyage n'est pas l'observation. (p. 327)

Et parfois, en s'arrêtant trop tard, d'épancher son amour :

17 avril 1777 - Séance chez Zoffani. Le portrait sera charmant et cette forme est mille fois plus agréable que la miniature sur une boîte ou la tête seule de grandeur naturelle. La figure sera entière, environ 15 pouces de hauteur, assise sous des arbres et une vue de Toscane dans l'éloignement. J'ai loué tout cela très sincèrement et sans imaginer que deux petits bras viendraient s'appuyer sur mes épaules pour me dire à l'oreille avec une voix charmante que le portrait serait pour moi, pour moi-même. Me voilà en possession de toutes les séances ; j'aurai voix chez le peintre, je serai chargé de regarder la physionomie de ma cousine, de quoi je m'acquitterai fort bien. Mais basta. Ceci est un journal. (p. 347)

Et s'il tombe malade et que Matilda vienne le veiller...

30 mai 1777 - Tante et cousine partagent leurs soins et leurs visites entre les malades. Il est au-dessus de la portée d'un journal de dire tout ce que ces soins-là ôtent de souffrances dans les peines et ajoutent de charmes aux plaisirs. (p. 366)

Pauvre journal ! on passe son temps à lui rappeler ses limites...

15 juillet 1777 - Une promenade à pied avec cousine Matilda. Comme le reste est digne d'oubli, je ne le ferai point passer dans mes écrits à l'immortalité. (p. 386). 
De ce reste, il ne parlera donc pas. Mais de la promenade elle-même, que dit-il d'autre, sinon qu'elle a eu lieu, et qu'elle n'est pas digne d'oubli ? Il la fait passer à l'immortalité, mais en allusion. Le journal est coincé entre l'insignifiant et l'indicible ! Et il n'est pas plus autorisé à exprimer la douleur que la joie. Quand on va être obligé, à Livourne, de quitter les nouveaux mariés, le déchirement sera suggéré ainsi :

4 septembre 1777 - Préparatifs et jeudi 4 départ de Livourne. Les gens peu touchés font des élégies sur le sujet de leur douleur; mais notre séparation d'avec les heureux époux que nous laissons ne doit pas être exposée pour le récit à la froideur d'un journal. (p. 395)

Deux raisons opposées sont données pour ne pas dire la douleur : la peur d'être... trop chaud («faire des élégies ») et soupçonné d'insincérité, la peur d'être trop froid, d'écrire dans le style factuel du journal. C'est par le biais d'une double prétérition que l'émotion finit par filtrer. Il ne s'agit plus là d'un problème de relations (cette douleur est honorable et partagée), mais de ton et de mesure. Le journal est plat et sec, il ne sait pas vibrer. Mais à l'idée d'un texte qui vibrerait, on prend peur. Entre la sécheresse et l'emphase, la voie d'un discours intime ne semble pas encore ouverte...

$\mathrm{Au}$ retour à Prangins, Louis-François reprend l'ancien journal qu'il va tenir jusqu'au départ des dames anglaises (22 novembre 1777) dans un registre familier sans être intime. Il suit la vie quotidienne, exprime parfois un sentiment de bonheur (22 septembre, 24 octobre), se permet une petite méchanceté (à propos d'une dame : « chose étrange, elle a été, dit-on, belle femme ", 19 octobre), raconte comme à l'accoutumée occupations, visites, lectures, spectacles - mais on le soupçonne de taire l'essentiel, qui va fuser au moment du départ. Comment, après plus d'un an d'amour et de vie quotidienne en commun, pouvoir supporter la séparation? Le 8 novembre, le voilà si enrhumé, et au lit, qu'il transmet la tenue du journal à... Matilda. Du 9 au 21 novembre (veille du départ), c'est elle qui tient la plume - service minimum, c'est rapide, sec, factuel - mais c'est elle aussi qui craque la première, car à ces notations factuelles se mêlent brusquement le monologue intérieur de sa douleur et la montée de l'angoisse (p. 425-426). Ce ne seront que quelques phrases - mais qu'il pourra, lui, relire après son départ. 15 novembre : «Ce départ est encore un peu renvoyé par un malheureux rhume qui vient très à propos. Faudra-t-il donc que le terme arrive?»17 novembre: "Convalescence de l'enrhumé. » 18 novembre : « Délai déterminé, mais le terme est fixé : ce sera samedi 22. » 21 novembre : "Quel jour, quel dernier jour...» Est-ce son cri du cœur à elle ? L'écriture sous dictée de son cri du cœur à lui ? C'est en tout cas l'un des deux seuls moments où l'on entende une parole directe, intime, non contrôlée. L'autre moment sera l'explosion du bonheur lors des retrouvailles. L'édition traduit par des points de suspension un long trait de plume qui se perd dans le vide de la page et de l'absence.

Une fois Matilda partie, Louis-François, entraîné par son exemple, dit lui aussi sa détresse : "Je ne puis rendre compte de rien ce jour-là » (22 novembre), « Mon cœur est rempli et ma tête confusément occupée et travaillée " (23 novembre), et on le sent au bord du lyrisme, puisqu'il peut écrire : "Promenade à pied pour revenir à la maison sans avoir à la regarder comme une prison » (25 novembre), ce qui est presque «Un seul être vous manque... ». Puis tout se calme et le journal reprend son cours sec, factuel, et ses distances. Le $1^{\mathrm{er}}$ décembre, anniversaire de ses 36 ans, Louis-François se laisse pourtant aller à méditer sur le temps passé et sur l'expérience accumulée, mais il se reprend, et remet sèchement le journal à sa place (comme si son journal, indiscret, avait écouté aux portes de son cœur): "Laissons la vanité métaphysique. Rien n'est plus loin d'une relation jour par jour » (p. 429). La sécheresse du journal, puis son arrêt complet entre le 
$1^{\mathrm{er}}$ janvier et le 20 mars 1778, s'expliquent très simplement par la concurrence de la correspondance qui, elle, va bon train, on s'en doute, avec « les dames anglaises »:

20 mars 1778 - Le temps que je puis donner à écrire a été employé à la correspondance avec les Anglaises à Paris jusqu'au lundi 16 janvier et depuis à Londres jusqu'à ce jour. Ainsi je n'ai pas seulement un léger reproche à me faire d'inexactitude ou d'impersévérance. (p. 435)

23 avril 1778 - C'est un parti pris de passer à l'air presque tous les moments de jour dans les journées de beau temps. Ceci met obstacle à l'exactitude d'inscrire au journal. Mais la correspondance anglaise très fidèlement soutenue en détourne bien davantage. (p. 439)

Et lui-même, dans la dernière entrée de son journal de célibataire (25 avril 1778), constatera l'écart total qu'il y a entre sa vie, si remplie, et son journal, si vide !

\section{Troisième phase : du 2 août 1778 au 31 janvier 1779 (p. 441-476)} Louis-François a souci, dès son retour, de "remettre au niveau » le pauvre journal complètement abandonné pendant trois mois. La reprise se fait en deux temps : d'abord, de l'écriture de Matilda, mais sous la dictée de Louis-François, le récit de son voyage aller, de Prangins à Londres, pour retrouver Matilda (voyage du 27 avril au 20 mai, p. 441-442). Puis il reprend la plume, en précisant qu'on est le 2 août :

Je reprends le journal depuis cette date. L'intervalle entre le dimanche 26 avril et le dimanche 2 août sera rempli par une relation historique dont les mémoires sont encore dans ma tête et dans mon cœur. (p. 442)

Mais cette relation historique, dictée à Matilda, n'a pas été continuée au-delà du 20 mai. Pourquoi ? L'explication est simple. Tant qu'il raconte son voyage, c'est encore possible, quoiqu'on n'ait jamais vu voyage où il soit moins parlé du voyage ! C'est à la fois un pèlerinage (« elles » sont passées par là), une mission secrète (il ne doit pas dire où il va ni pourquoi) et une expédition de conquête... Le texte frôle le lyrisme en permanence, puis, à la fin, dérape et s'y abandonne carrément, l'espace de quelques lignes, où l'émotion déborde dans un style qui se rapproche de celui de La Nouvelle Héloïse :

Je vois Londres, je vais arriver Argyll Street, je suis à la porte, Mrs Guiguer, $\mathrm{n}^{\circ} 15 ;$ la voilà, je l'embrasse, elle me laisse monter au second étage. Ma chère cousine, me voici. Je ne sais plus le reste de la conversation, mais dois-je croire que je repartirai jamais en me séparant d'Elle ? Sans rien décider, il est très décidé que non. Ce jour était un samedi 20 mai. (p. 442)

Nous ne sommes pas là dans un journal, mais dans une "relation historique ", mais si présente qu'il y emploie le présent de narration, des phrases hachées, presque comme un monologue intérieur. Une poussée d'émotion fait craquer l'allure objective et factuelle du journal. Et Matilda, qui écrit sous la dictée de Louis-François, s'arrête là pour plusieurs raisons : l'essentiel est dit, ce n'est pas le lieu dans un journal d'écrire un roman lyrique, et la date sacrée des retrouvailles résume tout : elle est d'ailleurs écrite en caractère deux fois plus gros que le reste! Peut-être aussi la situation est-elle bizarre : ce récit d'amour est dicté à la personne qui en est l'objet...

Après cette mini-explosion, Louis-François entame un journal factuel, et très vite, le lendemain même, il abandonne la plume à Matilda. Cela ne signifie pas forcément qu'elle rédige elle-même, il est probable qu'elle écrit souvent sous sa dictée, surtout au début. C'est un mélange indiscernable des deux - un peu comme naguère avec Renz. C'est 
à la fois un partage affectif, et l'occasion d'une "mise au courant» de la gestion du domaine. On lit donc une chronique familière, des visites, des occupations, la vie quotidienne, des sorties, promenades, etc. La première personne, peu fréquente, désigne tantôt lui (il arrive alors que « je » soit souligné, ou traité comme un nom propre), tantôt elle (adjectifs au féminin). Le plus souvent, on reste dans le "nous», ou dans l'impersonnel (phrases nominales). Mais les nouvelles qu'elle donne périodiquement de sa santé montrent que, rapidement, le journal devient son affaire à elle. 13 septembre 1778 : "J'étais assez incommodée, ce qui dérange l'exactitude du présent journal. » 18 septembre: «Je me suis trouvée assez incommodée pour ne point paraître.» 19 septembre: «Je suis mieux et je vois le monde » (p. 453-455). Et finalement, cette demi-confidence :

3 octobre 1778 - Il a été fait une confidence à Madame de Ribaupierre, faite avec plaisir et reçue avec plaisir; mais nous ne faisons pas la même confidence au papier. (p. 458)

Formulation charmante, et curieuse !-Elle me rappelle un dessin humoristique de Mankoff, dans le New Yorker (19 juin 1989), où l'on voit un homme assis à une table, écrivant dans un cahier, et la légende dit : «Dear Diary : None of your damn business!» (que je traduis librement par : "Cher Journal, ceci n'est pas pour ton vilain nez! »). Il y a, bien sûr, contradiction. D'un côté, le journal n'est pas traité comme un ami, il est exclu de la sphère de l'intimité, on lui ferme la porte au nez. D'un autre côté, il est traité comme un ami, puisqu'on lui dit qu'on a fait une confidence à une amie, même si on ne lui dit pas à quel sujet, mais en soulignant à deux reprises que cette confidence a été faite et reçue avec plaisir ! Et visiblement, ce plaisir, on l'éprouve aussi à en faire confidence au papier, même si on en a un peu honte, honte qu'on exprime et dont on se venge en lui faisant une cachotterie! D'où cette figure de prétérition, qui dit tout par sa manière d'annoncer qu'elle ne dira pas. Le papier, qui est loin d'être bête, a deviné : Matilda est enceinte. Il a aussi deviné que lui-même va bientôt faire partie de la famille! Jusque-là relégué à l'office, il va entrer au salon, dans la chambre des enfants, dans la chambre conjugale. Il va devenir dame de compagnie, confident. Même si, pour l'instant, on le snobe encore un peu. Mais c'est une épreuve initiatique : il suffit d'attendre, son heure viendra.

Cette grossesse, un peu difficile, va interrompre le journal plus de deux mois, du 18 octobre au 25 décembre 1778. Elle ne sera jamais nommée directement, mais maintenant, on considère que le journal, un grand garçon, est au courant. On peut lui expliquer pourquoi on l'a négligé :

25 décembre 1778 - Il faut commencer par rendre compte des causes du silence du journal pendant cet intervalle. J'ai été gardé par le journaliste et je n'ai pu lui prêter ma main ; mais je veux oublier les temps d'incommodité et de maladie, bien loin d'en fixer la mémoire par une relation écrite. La joie de la santé n'en est que mieux éprouvée après deux mois de peine. (p. 463)

Même demi-mesure, mais cette fois l'exclusion du journal a une autre raison, qui implicitement l'inclut dans la sphère de l'intimité, du moins au bord. Ce n'est pas parce que c'est trop personnel, mais parce que ce serait trop pénible. En revanche, on veut bien lui faire part du rétablissement. Il a un pied dans la maison. Pendant deux longues pages, Matilda (et le journaliste) vont récapituler ce qui s'est passé de notable dans le domaine pendant ces deux mois, jusqu'à ce que, le dimanche 20 décembre, elle puisse enfin sortir, aller à l'église (« le monde nous est rouvert », p. 466) et reprendre le fil de sa chronique : «Le journal ayant été repris le samedi pour insérer en abrégé le nombre de semaines omises, nous le reprenons jour par jour » (p. 466). Cette chronique ne s'abstient pas de noter un rayon de soleil (samedi 26 décembre). Et puis, le journal étant au courant, on 
peut lui expliquer, le 2 janvier (p. 469), qu'on coupe «les premiers habillements qui ne doivent servir qu'à la fin de mai » (on précise ainsi le terme de la grossesse), ou les démarches pour retenir une nourrice, malgré des vues toutes rousseauistes sur la question (« Nous nous sommes décidés pour elle, quoiqu'en notre cœur nous ayons donné notre confiance à la nourrice naturelle », 10 janvier 1779, p. 471). Puisque le journal est fait pour commémorer non les peines, mais le bonheur, les fêtes données le 15 janvier pour l'anniversaire de Matilda seront rapportées par le menu, y compris la citation in extenso de vers accompagnant un bouquet, vers « auxquels on ne peut donner un meilleur éloge qu'en les transcrivant, puisqu'en les trouvant dans notre journal nous pourrons les relire si souvent » (p. 472).

31 Un des éléments de familiarité, à défaut d'intimité, du journal, vient de l'intrusion d'un dialogue entre les époux. Parfois, semble-t-il, Matilda " prête la main », et Louis-François dicte. Mais parfois elle écrit à son propre compte et peut même, gentiment, par taquinerie, profiter du fait qu'elle tient la plume pour manifester son désaccord avec son seigneur et maître :

19 janvier 1779 - Pendant la lecture du soir, Monsieur Joël propose de crayonner le seigneur du lieu qui se laisse faire quoique en bonnet de nuit, qui par hasard est encore sur sa tête en raison d'un rhume. Le crayon est très ressemblant quoi que ledit seigneur en puisse dire. (p. 473)

Le journal est « notre » journal (p. 472), jamais vraiment « mon journal », même si LouisFrançois est appelé souvent le "journaliste», en ce sens que c'est lui qui dirige l'entreprise. Mais ce "notre » évolue du collectif au familier. L'idée constante que le journal n'est pas fait pour l'expression de l'affectivité est battue en brèche par les situations de dialogue. C'était déjà le cas dans la première partie du journal, quand les deux journalistes faisaient des allusions à leurs différences. Le journal reste ouvert sur une petite communauté virtuellement plus large que le couple (Renz prend de nouveau la plume en janvier 1779 pour dresser une liste de lectures), mais il s'installe dans un ton familier, s'acclimate par bouffées à l'expression de sentiments, à la notation de sensations. L'impression qu'il donne de rôder autour de cela tient au système d'énonciation : la personne qui tient la plume a souvent recours à des périphrases, ou à la troisième personne, pour se désigner, et elle emploie souvent le «nous", ou des tournures impersonnelles. Devinez, par exemple, qui a annoncé la grossesse à Madame de Ribaupierre : «Il a été fait confidence... »!

Ce qu'on voit donc dans ce premier volume, c'est le glissement d'un « nous » officiel vers un « nous » familier, favorisé par un système d'écriture à deux, et le passage de l'amitié à l'amour. Attendons le volume II et la naissance annoncée pour voir sans doute un nouveau pas en avant dans la familiarité. Mais de ce «nous» familier, parfois étayé sur un « je » prudent, au journal intime, il y a encore un abîme.

\section{NOTES}

1. Louis-François Guiguer, baron de Prangins, Journal 1771-1779, édité et annoté par Rinantonio Viani, Introduction de Chantai de Schoulepnikoff, Prangins, Association des amis du Château de 
Prangins, 2007. Les deux autres volumes du Journal (1779-1784 et 1784-1786) ont été publiés respectivement en 2008 et 2009. J'ai, dans les citations, adopté l'orthographe moderne et régularisé la formulation des dates. Le manuscrit se trouve aux Archives Cantonales Vaudoises, à Lausanne.

\section{RÉSUMÉS}

Le volume I récemment publié du Journal Intime de Louis-François Guiguer, baron de Prangins, démontre que l'écriture d'un journal intime était encore une pratique visible, factuelle et quasiment publique au cours $d u \mathrm{XVIII}^{\mathrm{e}}$ siècle. Motivé par le désir d'écrire sur son amour pour Matilda (qu'il devait épouser plus tard) dans son propre journal intime, le jeune homme tergiverse et ruse avec cette forme indiscrète qui est faite pour tout sauf pour écrire sur l'intimité. « Journal intime » aurait été un terme contradictoire à cette époque.

The recently published volume I of Journal, by Louis-François Guiguer, Baron of Prangins, demonstrates that journal writing was still a visible, factual, virtually public practice during the eighteenth century. Motivated by the desire to write in his own journal about his love for Matilda, whom he would later marry, the young man prevaricates and uses cunning with this indiscreet form that is made for anything but writing about intimacy. "Personal journal" would have been a contradiction in terms during this era.

\section{INDEX}

Mots-clés : émergence de l'intimité, journal intime, dix-huitième siècle, censure, métadiscours Keywords : emergence of intimacy, diary, eighteenth century, censorship, metadiscourse

\section{AUTEUR}

\section{PHILIPPE LEJEUNE}

Association pour l'Autobiographie et le Patrimoine Autobiographique 